\title{
SoSyM reflections: the 2018 "State of the Journal" report
}

\author{
Huseyin Ergin ${ }^{1} \cdot$ Jeff Gray ${ }^{2} \cdot$ Bernhard Rumpe ${ }^{3} \cdot$ Martin Schindler $^{3}$ \\ Published online: 22 January 2019 \\ ๑) Springer-Verlag GmbH Germany, part of Springer Nature 2019
}

We are delighted to kick off another volume of SoSyM, with this first issue of 2019! The "health" of SoSyM continues to be in good shape, with evidence of its growing popularity and impact. The statistics reported in the next section indicate that we have a steady rise in the number of incoming and also accepted papers. Therefore, we are proud to announce that beginning with this issue, we will have an increase from four to six issues per year. This will allow us to grow the overall number of pages published each year.

However, we have two ongoing problems that we are focused on addressing over the next year.

First, our turnaround times for the reviews are often higher than we would like. This shows that SoSyM is a high-quality journal and reviewing is taken very seriously. Our goal is to reduce reviewing times; in particular, the time to identify appropriate reviewers, which seems to be the initial bottleneck experienced by our editors. Although the total number of days from submission to final decision has been reduced by $\sim 20 \%$ over the past year (now down to 138 days), we believe that we can still improve in this area. We thank all of the reviewers of the 2018 submitted papers. Each reviewer from 2018 is mentioned later in this editorial and received a certificate at the beginning of 2019 in recognition of our appreciation for their service to SoSyM.

The other ongoing problem has emerged from our success in the increase in the number of submitted and accepted papers, which has created a publication time backlog that is too long. The backlog accrues from the number of papers

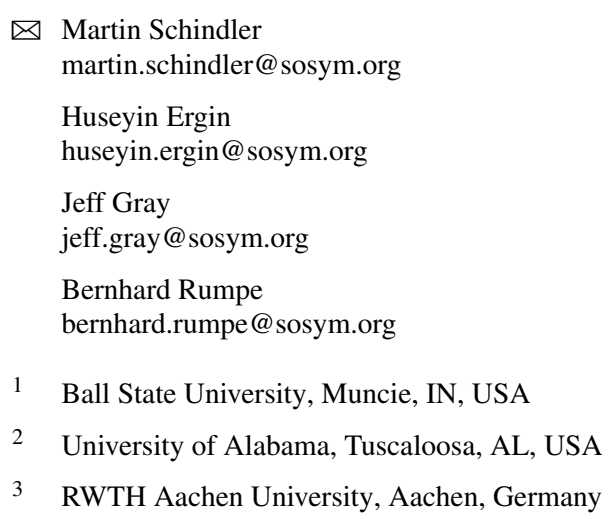

that has been accepted and already available online first, but not published in the printed version with a formal volume/issue/page number. Over the past 5 years, it has become apparent that the authors desire a formal bibliographic reference with volume and page numbers, primarily for the purpose of career evaluation. Those papers that are online first, but not formally published, often do not appear in frequently used citation indices (e.g., DBLP or Google Scholar). To address this concern, we have collaborated with our publisher (Springer) to identify new ways to handle the backlog. One effect of these discussions is the deep growth in the number of pages that will be printed (evident in this issue, which is the largest ever for SoSyM!) and the addition of two new issues per year (from four issues in the past, to six issues starting in 2019). We will keep the SoSyM community informed about the impact that these decisions make toward reducing the publication backlog.

\section{Summary statistics}

The four SoSyM issues published in 2018 contained 18 Regular papers, 21 Special Section papers, ten Theme Section papers, one Expert Voice, and five Guest Editorials. In total 1393 pages were published in volume 17.

We are very happy to report that the impact factor for SoSyM over the past year increased again to 1.722 (previously at 1.654 in 2017). This is the highest impact factor since the inception of SoSyM, which we believe offers evidence to the growing interest in software and systems modeling as a research discipline and to SoSyM's important role in the modeling research community. We also have observed a high number of paper downloads over the past year; in 2017, SoSyM papers were downloaded 73,352 times, which is roughly four times as high compared to the downloads in 2010 (there were 20,267 downloads in 2010).

The acceptance rate in 2018 was $22.1 \%$, which is a rather good number for a high-quality journal such as SoSyM. The average time to the final decision (accept or reject) has been reduced to 138 days. 


\section{SoSyM's ten-year most influential paper awards}

MODELS 2018, held in Copenhagen, Denmark, in October 2018, provided another opportunity to recognize the most influential SoSyM papers from the past 10 years during the banquet dinner. The selection was based on the ISI citation index among papers published in SoSyM 10 years ago (2008). More information about the awards can be found at: http://www.sosym.org/awards/.

The SoSyM 2018 "Ten-year most influential Regular paper award" was given to:

Colin Atkinson and Thomas Kühne, "Reducing accidental complexity in domain models", In: Journal on Software and Systems Modeling (SoSyM), Volume 7, Issue 3, pp. 345-359, Springer, July 2008. https://doi. org/10.1007/s10270-007-0061-0

The SoSyM 2018 "Ten-year most influential Theme Section paper award" was given to:

Jürgen Dingel, Zinovy Diskin, and Alanna Zito, "Understanding and improving UML package merge", In: Journal on Software and Systems Modeling (SoSyM), Volume 7, Issue 4, pp. 443-467, Springer, October 2008. https://doi.org/10.1007/s10270-007-0073-9

\section{SoSyM's Journal-First papers at MODELS 2018}

We now have established a continuous collaboration between SoSyM and the MODELS conference in organizing the SoSyM "Journal-First" opportunity, which enables authors of recent SoSyM papers to present their work across the core conference sessions at MODELS. Through this collaboration, selected SoSyM authors have the opportunity to reach a broader audience to present their work. This also benefits the MODELS conference program by including research talks that explore more depth through analytical and empirical evidence than can be presented in a traditional conference submission. The MODELS 2018 program featured four articles that were accepted during the previous year (from July 2017 through June 2018). The SoSyM “Journal-First” papers presented at MODELS 2018 were the following:

- Alexandre Petrenko, "Toward testing from finite state machines with symbolic inputs and outputs", In: Journal on Software and Systems Modeling (SoSyM), https://doi. org/10.1007/s10270-017-0613-x, Springer. 2017.
- Fabio Cremona, Marten Lohstroh, David Broman, Edward A. Lee, Michael Masin, and Stavros Tripakis, "Hybrid co-simulation: It's about time", In: Journal on Software and Systems Modeling (SoSyM), https://doi.org/10.1007/ s10270-017-0633-6, Springer. 2017.

- Hugo Bruneliere, Erik Burger, Jordi Cabot, and Manuel Wimmer, "A feature-based survey of model view approaches", In: Journal on Software and Systems Modeling (SoSyM), https://doi.org/10.1007/s10270-017-06229, Springer. 2017.

- Markus Voelter, Bernd Kolb, Klaus Birken, Federico Tomassetti, Patrick Alff, Laurent Wiart, Andreas Wortmann, and Arne Nordmann, "Using language workbenches and domain-specific languages for safety-critical software development", In: Journal on Software and Systems Modeling (SoSyM), https://doi.org/10.1007/s10270018-0679-0, Springer. 2018.

\section{Geri georg retires as SoSyM Assistant Editor}

For many years, the SoSyM community of authors, reviewers, and editors has corresponded and sought assistance from Geri Georg, one of our two Assistant Editors since the inception of the journal organization in 1999. Geri and our dearly departed colleague and co-founding Editor-in-Chief, Robert France (who passed away far too early in 2015 from cancer), concentrated for over a decade on the Regular papers, while Bernhard Rumpe and Martin Schindler focused on the Special and Theme issues, as well as Expert Voice papers. Geri's devotion to SoSyM was evident in the deep commitment she made to help authors with questions and to tirelessly maintain the "pulse" of the review process by assisting editors and reviewers with the deadline schedules of the review of Regular papers.

Geri has decided to retire from this long-lasting and deep responsibility of organizing the journal, in order to concentrate fully on her academic interests and pursuit of her hobbies, namely, traveling and photography!

In the names of all editors, reviewers, and authors, we thank Geri very much for all of her passionate service and work for SoSyM over the past two decades. We wish her well in all of her future pursuits in life! 

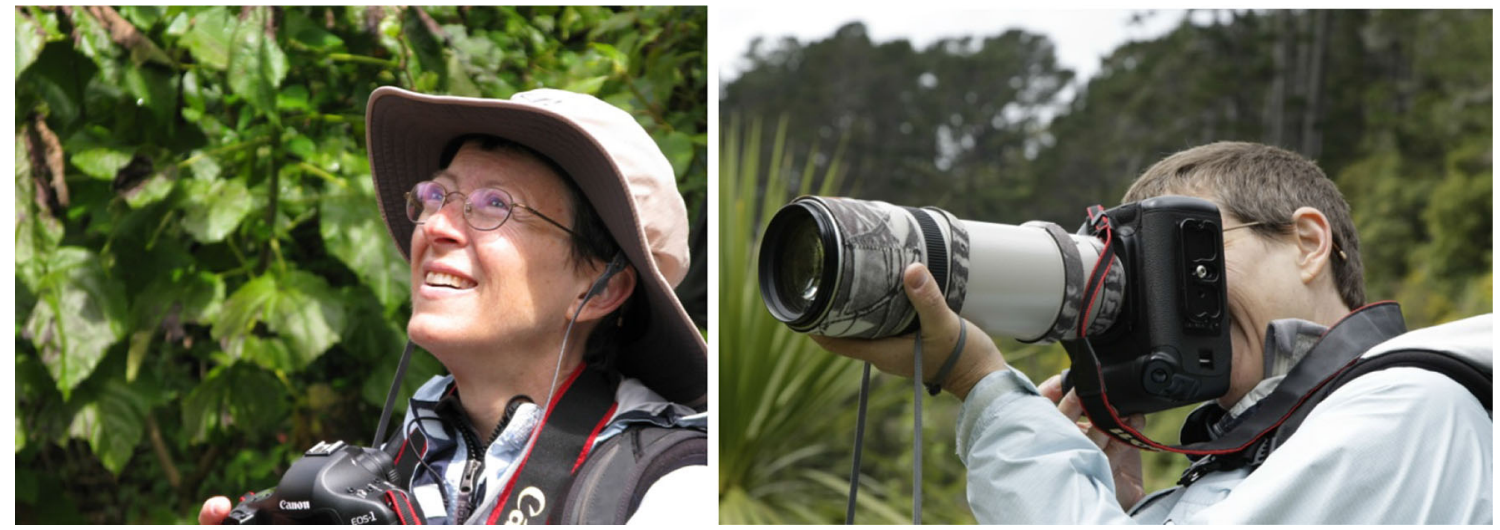

Geri Georg in New Zealand at MODELS 2011 (photos: Martin Schindler)

\section{Introducing Huseyin Ergin as SoSyM's new Assistant Editor}

We welcome Huseyin Ergin as the new Assistant Editor for SoSyM. Huseyin will help Jeff Gray with needs arising from the editorial process associated with Regular papers. For a seamless continuation of the journal, Geri and Huseyin have been collaborating since mid-2018 to help him prepare for the transition to his new role. Effective November 2018, Huseyin has taken over the full-time duties of Assistant Editor for SoSyM.

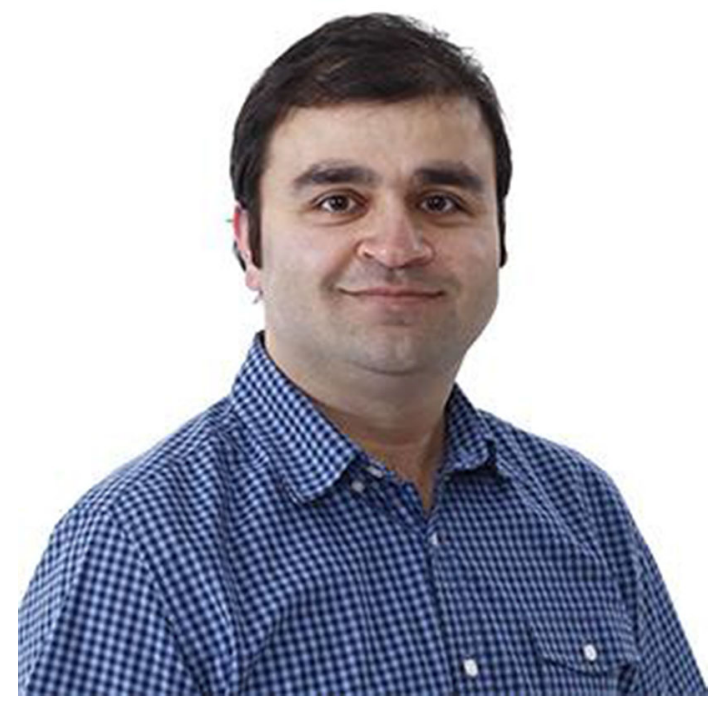

Huseyin is an Assistant Professor in the Department of Computer Science at Ball State University (located in Muncie, Indiana, the USA). Originally from Turkey, Huseyin's undergraduate degree in computer science is from Yeditepe University in Istanbul. He received his PhD in 2017 from the University of Alabama for his work on design patterns for model transformations. Additional biographical information about Huseyin and details about his research interests can be found at: http://www.cs.bsu.edu/homepages/ hergin/

We offer Huseyin a warm welcome, with anticipated success and enjoyment in this new role!

Message from Huseyin

When I was offered the position of Assistant Editor in mid2018, I did not need to think twice and immediately accepted the honor to serve SoSyM. I love the energy and welcoming spirit of the modeling community and am very happy and excited about many opportunities to engage with everyone through my new role. During the past 6 months, I learned so much from Geri (thanks Geri, you are the best!) about how I can help authors and editors in the most meaningful way possible. From now on, please consider me as your humble assistant and feel free to reach out to me for any kind of question or feedback!

\section{Changes to the Editorial Board}

Two of our long-time Editors have recently retired from the Editorial Board. We appreciate Hassan Gomaa and Roel Wieringa for all of their support and willingness to help with the SoSyM editorial responsibilities! Several new Editorial Board members will be announced throughout the year.

\section{Reviewers in 2018}

Below is the list of those who reviewed one or more papers for the journal in the last year. The complete list of reviewers can also be found on our website http://www.sosym.org/people/. We appreciate all of the help that the reviewers provided in service to the modeling community! 
Alain Abran, Abedallah Zaid Abualkishik, Mathieu Acher, Michael Adams, Naci Akkøk, Omar Alam, Aldeida Aleti, Perry Alexander, Mauricio Alferez, Shaukat Ali, Hamoud Aljamaan, Andrew Allen, Joao Paulo Almeida, Vander Alves, Daniel Amyot, Dimosthenis Anagnostopoulos, Eric Andonoff, Anthony Anjorin, Adil Anwar, Myla Archer, Danilo Ardagna, Ritu Arora, Said Assar, Evan Austin, Ahmed Awad, Jean-Philippe Babau, Mehdi Bagherzadeh, Arosha Bandara, Purushotham Bangalore, Earl Barr, Nicolas Belloir, Gabor Bergmann, Daniel Berry, Kahina Bessai, Karsten Boehm, Kirill Bogdanov, Artur Boronat, Paolo Bottoni, Johann Bourcier, Erwan Bousse, Ivona Brandic, Lars Brehm, Ruth Breu, Achim Brucker, Hugo Bruneliere, Paul Brunet, Andrea Burattin, Erik Burger, Loli Burgueño, Alexandru Butean, Cristina Cabanillas, Radu Calinescu, Trevor Carlson, Michel R. V. Chaudron, Antonio Cicchetti, Federico Ciccozzi, Tony Clark, Peter Clarke, Loek Cleophas, Rolland Colette, Karin Coninx, Jonathan Corley, Vittorio Cortellessa, Bernard Coulette, Luis Couto, Xavier Crégut, Christophe Cruz, Andrea D'Ambrogio, Fabiano Dalpiaz, Maya Daneva, Nancy Day, Rogerio de Lemos, Pierre de Saqui-Sannes, Julien DeAntoni, Thomas Degueule, Onur Demirors, Joerg Desel, Jack Deslippe, Antinisca Di Marco, Giovanna Di Marzo Serugendo, Davide Di Ruscio, J. Diaz-Pace, Juergen Dingel, Zinovy Diskin, Dulce Domingos, Heiko Dörr, Yanhua Du, Eric Dubois, Alexander Egyed, Andre Takeshi Endo, Huseyin Ergin, Gidon Ernst, Neil Ernst, Tulga Ersal, Huascar Espinoza, Dirk Fahland, Paolo Falcarin, João Faria, Anna Rita Fasolino, Michael Felderer, Michael Fellmann, Nicolas Ferry, Peter Fettke, Kathrin Figl, Andrew Forward, Ulrich Frank, Piero Fraternali, Erik Fredericks, Antonio García-Domínguez, Vahid Garousi, Geri Georg, Sepideh Ghanavati, Mohamad Gharib, Sudipto Ghosh, Fáber Giraldo, Mario Gleirscher, Claude Godart, Claudio Gomes, Abel Gómez, Carlos González, Mark Grebe, Heerko Groefsema, Alicia Grubb, Sérgio Guerreiro, Giancarlo Guizzardi, Jens Gulden, Stefan Hallerstede, Ragnhild Halvorsrud, Mark Harman, Thomas Hartmann, Jameleddine Hassine, Xiao He, Regina Hebig, Thomas Heinze, Maritta Heisel, Rogardt Heldal, Sebastian Herzig, Lom Messan Hillah, Georg Hinkel, Jennifer Horkoff, Mahmood Hosseini, Muhammad Zohaib Iqbal, Fuyuki Ishikawa, Ferosh Jacob, Manfred A. Jeusfeld, Lingxiao Jiang, Mohamed Jmaiel, Haruhiko Kaiya, Anna Kalenkova, Holger Karl, Gabor Karsai, Erdal Kayacan, Steven Kelly, Mickael Kerboeuf, Djamel Eddine Khelladi, Joerg Kienzle, Jacques Klein, Alexander Knapp, William Knottenbelt, David Knuplesch, Sahar Kokaly, Natalia Kokash, Shekoufeh Kolahdouz-Rahimi, Dimitrios Kolovos, Lamine Kone, Tomaz Kosar, Anne Koziolek, Padmanabhan Krishnan, Lars Michael Kristensen, John Krogstie, Akhil Kumar, Juliana Küster Filipe Bowles, Marcello La Rosa, Kevin Lano, Peter Gorm Larsen, Young Lee, Elizabeth Leonard, Henrik
Leopold, Timothy Lethbridge, Emmanuel Letier, Shuai Li, David Lo, Malte Lochau, Francesco Longo, Roberto LopezHerrejon, Peri Loucopoulos, Levi Lucio, Ivan Lukovic, Ioanna Lytra, Narciso Martí-Oliet, Atif Mashkoor, Aaron Massey, Raimundas Matulevicius, Tanja Mayerhofer, Mark McKelvin, Julio Medina, Claudio Menghi, Stephan Mennicke, Andreas Metzger, Zoltan Micskei, Felix Mohr, Ana I. Molina, Pedro Molina, Leonardo Montecchi, Mirko Morandini, Jean-Marie Mottu, Haralambos Mouratidis, Chokri Mraidha, Adam Murray, John Mylopoulos, Safia NaitBahloul, Himanshu Neema, Shiva Nejati, Bernd Neumayr, Michael Nieke, Mara Nikolaidou, Erik Gøsta Nilsson, Jens Nimis, Emmanuel Nowakowski, Markus Nüttgens, Ileana Ober, Iulian Ober, Saahil Ognawala, Dusan Okanovic, Peter Ölveczky, Aida Omerovic, Andreas Opdahl, Richard Freeman Paige, Jose Ignacio Panach, David Parker, Jeffrey Parsons, Oscar Pastor, Fabrizio Pastore, Jose Osvaldo Saldanha Paulino, Jan Peleska, Barbara Pernici, Dorina Petriu, Holger Pfeifer, Alfonso Pierantonio, Raquel Pillat, Geert Poels, Fiona Polack, Gregor Polancic, Saul E. Pomares, Roman Popp, Jose Proenca, Erik Proper, Elke Pulvermueller, Paul Ralph, Istvan Rath, Bendraou Reda, Raghu Reddy, Manfred Reichert, Hajo Reijers, Iris Reinhartz-Berger, Guizzardi Renata, José Matías Rivero, Roberto Rodríguez-Echeverría, Pascal Roques, Matteo Rossi, Nicolas Rouquette, Francisco Ruiz, Rick Salay, Jesus Sánchez-Cuadrado, Axel Scheithauer, Rainer Schmidt, Pierre-Yves Schobbens, Carl Schultz, Dennis M. M. Schunselaar, Ed Seidewitz, Christoph Seidl, Emil Sekerinski, Laura Semini, Anas Shatnawi, Sofia Sherman, Samira Si-Said Cherfi, Michael Sievers, Bruno Silva, Jocelyn Simmonds, Monique Snoeck, Mark Snyder, Pnina Soffer, Oleg Sokolsky, Stéphane Somé, Hui Song, Thierry Soriano, Jean-Sebastien Sottet, Stefan Stanciulescu, Martin Steffen, Matthew Stephan, Perdita Stevens, Janis Stirna, Daniel Strüber, Arnon Sturm, Meng Sun, Gerson Sunye, Gabriele Taentzer, Casper Thule, Matthias Tichy, Massimo Tisi, Christos Tsigkanos, Benjamin Tyler, Zoltán Ujhelyi, Antonio Vallecillo, Nick R. T. P. van Beest, Mark van den Brand, Simon Van Mierlo, Hans Vangheluwe, Juan Manuel Vara, Sira Vegas, Eric Walkingshaw, Marco Wehrmeister, Matthias Weidlich, Mathias Weske, Bernhard Westfechtel, Danny Weyns, Manuel Wimmer, Jim Woodcock, Andreas Wortmann, Kim Wuyts, Huafeng Yu, Tao Yue, Anna Zamansky, Jelena Zdravkovic, Gefei Zhang, and Wei Zhang.

\section{Contents of this Issue}

This issue is one of the largest ever published by SoSyM, with a Theme Section, Special Section, invited Overview paper, and ten Regular papers. The contents of this issue include the following: 
1. Theme Section on Model-Driven Engineering for Component-Based Software Engineering

Guest Editors: Jan Carlson, Federico Ciccozzi, Patrizio Pelliccione, and Massimo Tivoli

2. STAF 2015 Special Section

Guest Editors: Alfonso Pierantonio, Jasmin Blanchette, Francis Bordeleau, Nikolai Kosmatov, Gabi Taentzer, and Manuel Wimmer

\section{Overview paper}

- "A feature-based classification of formal verification techniques for software models" by Sebastian Gabmeyer, Petra Kaufmann, Martina Seidl, Martin Gogolla, and Gerti Kappel

\section{Regular Papers}

- "Effective product-line testing using similarity-based product prioritization" by Mustafa Al-Hajjaji, Thomas Thüm, Malte Lochau, Jens Meinicke, and Gunter Saake

- "A model-driven approach for vulnerability evaluation of modern physical protection systems" by Annarita Drago, Stefano Marrone, Nicola Mazzocca, Roberto Nardone, Annarita Tedesco, and Valeria Vittorini

- "A metrics suite for UML model stability" by Amjad Abu Hassan and Mohammad Alshayeb

- "Lessons learned from developing mbeddr: A case study in language engineering with MPS" by Markus Voelter, Bernd Kolb, Tamás Szabó, Daniel Ratiu, and Arie van Deursen
- "Realizing strategic fit within the business architecture: The design of a Process-Goal Alignment modeling and analysis technique" by Ben Roelens, Wout Steenacker, and Geert Poels

- "DataMock: An Agile Approach for Building Data Models from User Interface Mockups" by José Matías Rivero, Julián Grigera, Damiano Distante, Francisco Montero, and Gustavo Rossi

- "Reusable specification templates for defining dynamic semantics of DSLs" by Ulyana Tikhonova

- "Interactive web interfaces modeling, simulation and analysis using Colored Petri Nets" by Taffarel BrantRibeiro, Rafael Araújo, Igor Mendonça, Michel Soares, and Renan Cattelan

- "Synthesis and exploration of multi-level, multiperspective architectures of automotive embedded systems" by Jordan Ross, Alexandr Murashkin, Jia Hui Liang, Michal Antkiewicz, and Krzysztof Czarnecki

- "SQL-PL4OCL: An automatic code generator from OCL to SQL procedural language" by Marina Egea and Carolina Dania

We wish you a Happy New Year with the hope that you enjoy reading the papers in this issue!

Huseyin Ergin, Jeff Gray, Bernhard Rumpe, and Martin Schindler

Publisher's Note Springer Nature remains neutral with regard to jurisdictional claims in published maps and institutional affiliations. 